\title{
On the Influence of the Mean-Free-Path Parameter on Intranuclear Cascade Calculations.
}

\author{
J. J. Hogan and K. I. Burns \\ Department of Chemistry, MeGill University - Montreal, Canada \\ E. Gadioli, E. Gadioli Erba and G. Tagliaferri \\ Istituto di Scienze Fisiche dell'Università - Milano \\ Istituto Nazionale di Fisica Nucleare - Sezione di Milano \\ (Lett. Nuovo Cimento, 23, 89 (1978))
}

Page 89 , 10th row from the top. Instead of "energy integrated spectra" one should read "angle integrated spectra".

Page 91. Last row of table $I$, the reaction cross-section calculated using the short $\mathrm{mfp}$. Instead of $1210 \mathrm{mb}$ one should read $1910 \mathrm{mb}$.

Page 92. 3th row from the bottom. Instead of "We geel entitled ..." one should read «We feel entitled ...».

\footnotetext{
(C) by Società Italiana di Fisica Proprieta letteraria riservata

Direttore responsabile: CARLO CASTAGNOLI

Stampato in Bologna dalla Tipografla Compositori coi tipi della Tipografia Monograf Questo fascicolo è stato licenziato dai torchi il 30 -XI-1978
} 